\title{
Sequentially appearing erythema nodosum, erythema multiforme and Henoch-Schönlein purpura in a patient with Mycoplasma pneumoniae infection: a case report
}

\author{
Masaki Shimizu* ${ }^{1 *}$, Yasuhito Hamaguchi ${ }^{2}$, Takashi Matsushita², Yasuhisa Sakakibara ${ }^{1}$ and Akihiro Yachie ${ }^{1}$
}

\begin{abstract}
Introduction: A wide variety of skin manifestations are associated with Mycoplasma pneumoniae infection. However, the precise mechanisms by which M. pneumoniae infection is able to produce a variety of cutaneous manifestations are poorly understood.

Case presentation: An 8-year-old Japanese girl presented with sequential skin manifestations, including erythema nodosum, erythema multiforme and Henoch-Schönlein purpura. Although a chest radiograph showed no significant lung abnormalities, serological examinations revealed that these skin manifestations were associated with M. pneumoniae infection.

Conclusion: It has been reported that the variations in cutaneous manifestations of $M$. pneumoniae infection can be attributed to the immaturity of the adaptive immunity of a host. However, the case presented herein indicates that skin manifestations might not be specific for each individual. An awareness of the varied patterns of cutaneous disease is essential for the early diagnosis and treatment of patients with manifestations of M. pneumoniae infection.
\end{abstract}

Keywords: Mycoplasma pneumonia, Erythema nodosum, Erythema multiforme, Henoch-Schönlein purpura

\section{Introduction}

A wide variety of skin manifestations are associated with Mycoplasma pneumoniae infection [1]. It has been reported that the variations in cutaneous manifestations of M. pneumoniae infections can be attributed to the immaturity of the adaptive immunity of a host [2]. However, the precise mechanisms by which $M$. pneumoniae infection is able to produce a variety of cutaneous manifestations are poorly understood. In this report, we describe the case of a patient with sequentially appearing skin manifestations, including erythema nodosum, erythema multiforme and Henoch-Schönlein purpura, associated with $M$. pneumoniae infection.

\footnotetext{
* Correspondence: shimizum@staff.kanazawa-u.ac.jp

'Department of Pediatrics, School of Medicine, Institute of Medical, Pharmaceutical, and Health Sciences, Kanazawa University, 13-1 Takaramachi, Kanazawa 920-8641, Japan

Full list of author information is available at the end of the article
}

\section{Case presentation}

A previously healthy 8-year-old Japanese girl was referred to our hospital with a 2-day history of symmetrical, multiple, round, light pink, tender erythematous nodules on her lower legs and arthralgia of her ankles 13 days before admission (Figure 1). Their sizes were approximately $2 \mathrm{~cm}$. The diagnosis of erythema nodosum was made. The lesions gradually improved and disappeared 7 days before admission; however, the patient then developed multiple maculopapular and targetoid lesions over her lower legs 6 days before admission (Figure 2A). No involvement of the mucous membranes was found. The diagnosis of erythema multiforme was made. The physical examination also revealed tonsillitis. Specific serum immunoglobulin M (IgM) antibodies for $M$. pneumoniae were positive, and clarithromycin therapy $(300 \mathrm{mg} /$ day $)$ was started. The lesions gradually improved and disappeared 3 days before admission

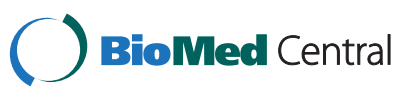




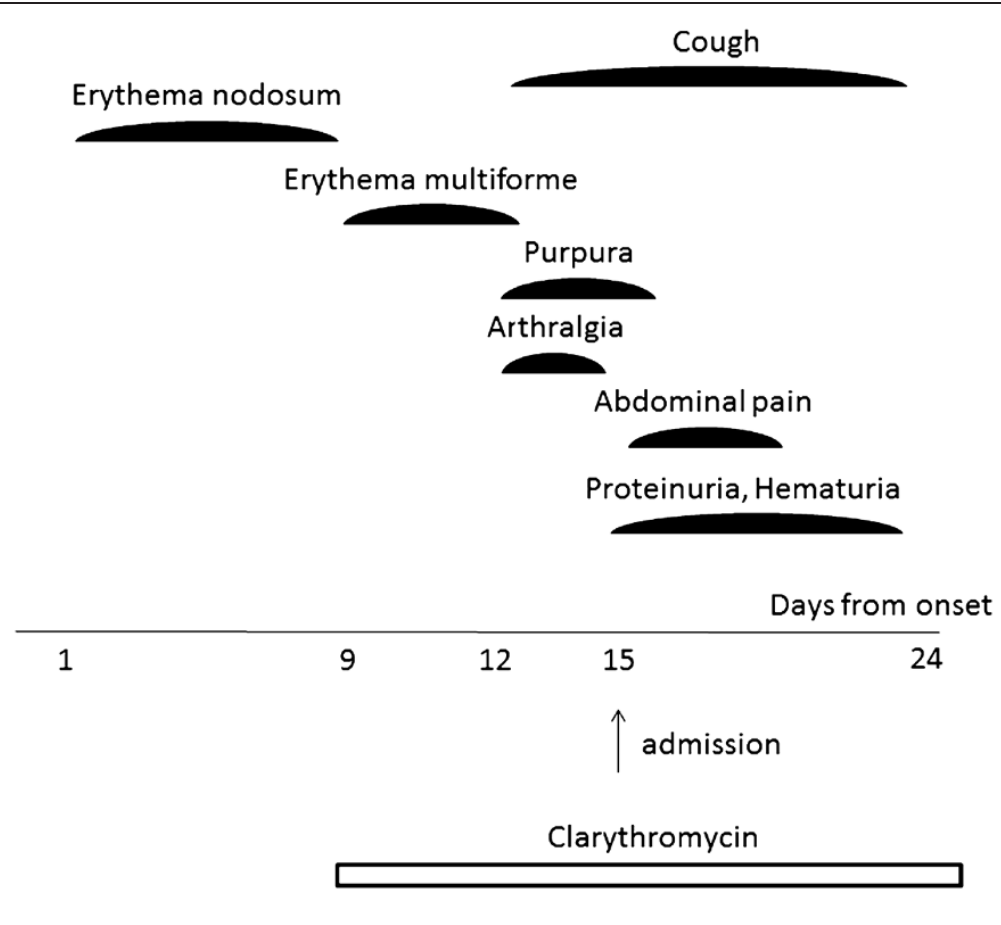

Figure 1 Clinical course.

(Figure 1). The patient had a mild cough; arthralgia of both hands; painful, localized edema on her scalp bilaterally; and a purpuric rash over her hip and lower legs concurrently 3 days before admission (Figure 2B). She also had abdominal pain and was admitted to our hospital. She was born in Japan and had not traveled out of Japan. She had no animal or insect bites. On admission, her body temperature was $36.8^{\circ} \mathrm{C}$, heart rate

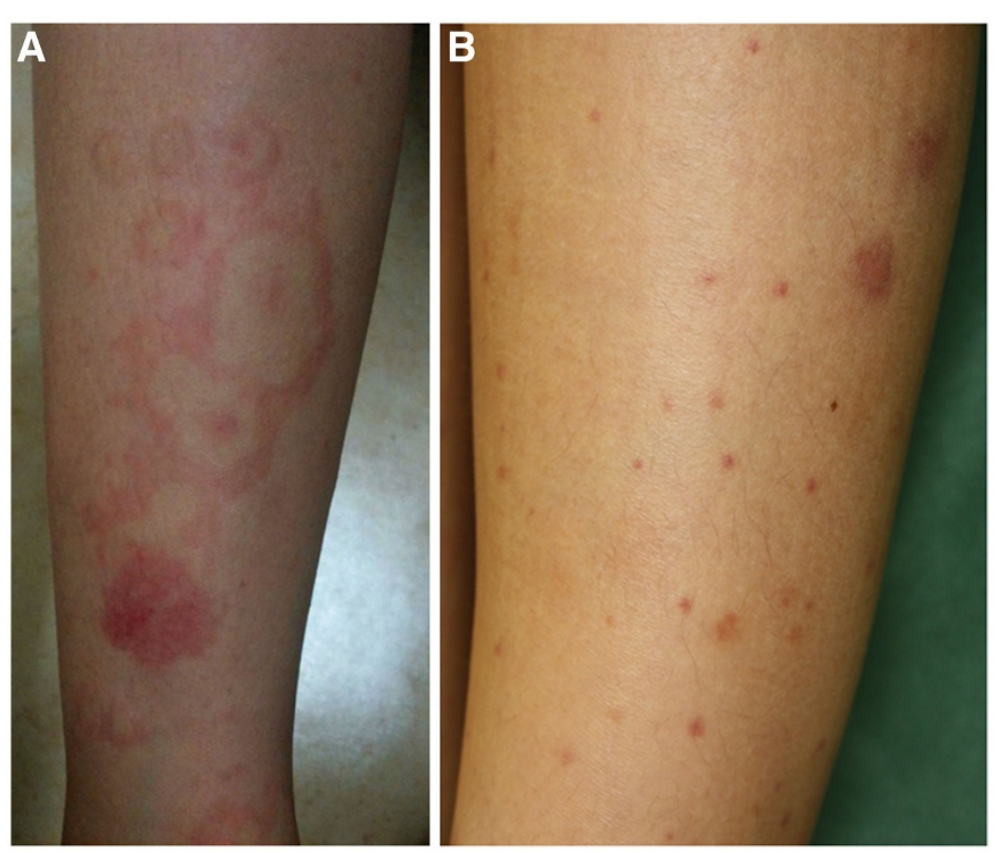

Figure 2 The cutaneous manifestations of Henoch-Schönlein purpura in our patient. (A) Erythema multiforme. Multiple papular, macular and target lesions of the right lower leg 6 days before admission. (B) Henoch-Schönlein purpura. Purpuric rash over the right lower leg was observed on admission. 
106 beats/minute and blood pressure 96/50mmHg. She had no weight loss. She had a purpuric rash over her hip and lower legs. The remainder of the examination was normal. Laboratory examinations showed a slightly elevated C-reactive protein level of $0.5 \mathrm{mg} / \mathrm{dl}$ (normal $<0.4 \mathrm{mg} / \mathrm{dl}$ ), white blood cell count of $8.44 \times 10^{9} / \mathrm{L}$, hemoglobin of $13.0 \mathrm{~g} / \mathrm{dl}$; platelet count of $220 \times 10^{9} / \mathrm{L}$, normal liver and kidney function, and urinalysis with $2+$ protein and $1+$ blood. Complement studies showed normal C3 of $119 \mathrm{mg} / \mathrm{dl}$ (normal, $86 \mathrm{mg} / \mathrm{dl}$ to $160 \mathrm{mg} / \mathrm{dl}$ ), C4 of $22 \mathrm{mg} / \mathrm{dl}$ (normal, $17 \mathrm{mg} / \mathrm{dl}$ to $45 \mathrm{mg} / \mathrm{dl}$ ) and slightly elevated $\mathrm{CH} 50$ of $59 \mathrm{U} / \mathrm{ml}$ (normal, $30 \mathrm{U} / \mathrm{ml}$ to $45 \mathrm{U} / \mathrm{ml}$ ). A culture from the throat revealed normal flora only. The test for anti-streptolysin $\mathrm{O}$ antibody was negative. Hepatitis B surface antigen and hepatitis C antibody were negative. The titer of complement fixation tests for M. pneumoniae was 1:20480. Chest radiograph revealed no significant lung abnormalities. On the basis of the appearance of the latest rash and the associated symptoms, the diagnosis of Henoch-Schönlein purpura was made. The patient was treated with clarithromycin $(300 \mathrm{mg} /$ day) for 3 weeks. Her symptoms improved gradually and disappeared 2 weeks later.

\section{Discussion}

A wide variety of cutaneous manifestations have been described (Table 1). The most common manifestation of M. pneumoniae infection is the exanthematous or maculopapular eruption. This reaction occurs in of $8 \%$ to $33 \%$

Table 1 Skin manifestations associated with Mycoplasma pneumoniae infection

\begin{tabular}{ll}
\hline Skin manifestation & Frequency \\
\hline Common & \\
Exanthematous skin eruptions & $8 \%$ to $33 \%$ \\
Erythema nodosum & $8 \%$ \\
Urticaria & $7 \%$ \\
Stevens-Johnson syndrome & $1 \%$ to $5 \%$ \\
Rare & \\
Bullous erythema multiforme & \\
Pityriasis rosea & \\
Henoch-Schönlein purpura & \\
Toxic epidermal necrolysis & \\
Kawasaki disease & \\
Subcorneal pustular dermatosis & \\
Thrombotic thrombocytopenic purpura & \\
Sweet's syndrome & \\
Raynaud's phenomenon & \\
Reiter syndrome & Urticarial vasculitis \\
Gianotti-Crosti syndrome &
\end{tabular}

of cases [1]. Erythema nodosum, urticaria and StevensJohnson syndrome are noted most often. The remaining cutaneous manifestations are rare [2].

The precise mechanisms by which $M$. pneumoniae produces a variety of cutaneous manifestations are poorly understood. Most cutaneous lesions are thought to be caused by the host response to antigens on these microbes rather than by the organisms themselves. Host immune responses such as immune complex-mediated injury, cytotoxic T-cell-mediated immune responses and autoimmune reactions have been speculated to play a crucial role in the development of cutaneous manifestations in patients with M. pneumoniae infection [3]. Patients with Henoch-Schönlein purpura likely have IgA-containing circulating immune complexes. Cytotoxic $\mathrm{T}$ cells have been implicated in patients with widespread vasculitis, such as in those with Kawasaki disease. Kano et al. [4] reported the cases of three patients with M. pneumoniae infection in a single family, each of whom manifested erythema nodosum, purpura and acute urticaria. They hypothesized that the variations in cutaneous manifestations of $M$. pneumoniae infections can be attributed to the immaturity of the adaptive immunity of a host. A feature of the present case is that different skin manifestations were observed in a single patient during the course of $M$. pneumoniae infection. This indicates that the type of cutaneous manifestation might not correlate with the maturity of the immune system in a given individual.

The most common cutaneous manifestations of $M$. pneumoniae infection, exanthematous or maculopapular eruption, may be either localized or confluent. These eruptions are self-limited, and no therapy for the cutaneous eruption is needed. Erythema nodosum is a reaction pattern caused by a variety of infections, including M. pneumoniae. In a previous report, it was shown that M. pneumoniae was the cause in $8 \%$ of patients with erythema nodosum [5]. Erythema multiforme is less common in M. pneumoniae infection. Thirty-five cases have been reported in the literature [6,7]. As shown in Figure $2 \mathrm{~A}$, patients with bullous erythema multiforme show target lesions, predominantly on the extremities, which spread to the trunk. Mucous membrane involvement occurs in most cases. Treatment includes topical corticosteroids for cutaneous lesions, local pain control with agents such as lidocaine solution, topical gel applied to painful oral lesions and treatment with appropriate antibiotics. M. pneumoniae-related Henoch-Schönlein purpura is a rare complication. A potential complication of M. pneumoniae infection and Henoch-Schönlein purpura is glomerulonephritis. Twenty-four cases of Henoch-Schönlein purpura-associated nephritis without skin findings have been related to M. pneumoniae infection [8]. Our patient is the first case with mild 
glomerulonephritis with skin lesions caused by $M$. pneumoniae infection. Fortunately, glomerulonephritis in our patient was mild. However, it is a serious internal complication of $M$. pneumoniae infection that should be screened for both during and after active infection. The underlying condition should be treated as appropriate with immunosuppressive agents, such as oral prednisone, if necessary.

It is still unclear whether drug ingestion contributes to skin reactions in M. pneumoniae infections. It has been reported that $17(60 \%)$ of 29 M. pneumoniae-infected patients received antibiotics prior to the development of exanthema [9]. In a study comparing azithromycin with erythromycin or amoxicillin/clavulanate, eruption was seen in $6.1 \%$ of patients taking azithromycin/clavulanate compared with $12.3 \%$ in those receiving erythromycin. Of the total group examined, $29.5 \%$ had M. pneumoniae infection [10]. It is possible that the combination of $M$. pneumoniae infection and drug exposure could increase sensitivity to the development of drug exanthemas.

The case of our patient emphasizes the importance of considering M. pneumoniae infection in the differential diagnosis of a patient with a wide variety of skin manifestations. M. pneumoniae infection is frequently considered in the differential diagnosis of patients with respiratory illnesses. This contrasts with patients who present with non-respiratory symptoms in the context of a recent or current unrecognized M. pneumoniae infection, for whom this pathogen is rarely considered in the initial differential diagnosis. An awareness of the varied patterns of cutaneous disease is essential for the early diagnosis and treatment of patients with manifestations of M. pneumoniae infection. Skin manifestations could appear even in cases without pneumonia, such as in our patient. A chest radiograph alone is not sufficient to make the diagnosis. Serological examinations are recommended.

\section{Conclusion}

We report the case of a patient with sequentially appearing skin manifestations, including erythema nodosum, erythema multiforme and Henoch-Schönlein purpura associated with M. pneumoniae infection. An awareness of the varied patterns of cutaneous disease is essential for the early diagnosis and treatment of patients with manifestations of $M$. pneumoniae infection.

\section{Consent}

Written informed consent was obtained from the patient's legal parent for publication of this manuscript and any accompanying images. A copy of the written consent is available for review by the Editor-in-Chief of this journal.

\section{Competing interests}

The authors declare that they have no competing interests.

\section{Authors' contributions}

MS wrote the manuscript and was responsible for literature research. YH, TM, YS and AY were involved in critically revising the manuscript for important intellectual content. All authors read and approved the final manuscript.

\section{Author details}

${ }^{1}$ Department of Pediatrics, School of Medicine, Institute of Medical, Pharmaceutical, and Health Sciences, Kanazawa University, 13-1 Takaramachi, Kanazawa 920-8641, Japan. ${ }^{2}$ Department of Dermatology, School of Medicine, Institute of Medical, Pharmaceutical, and Health Sciences, Kanazawa University, 13-1 Takaramachi, Kanazawa 920-8641, Japan.

Received: 4 June 2012 Accepted: 16 October 2012

Published: 23 November 2012

\section{References}

1. Copps SC, Allen VD, Sueltmann S, Evans AS: A community outbreak of Mycoplasma pneumoniae. JAMA 1968, 204:123-128.

2. Schalock PC, Dinulos JGH: Mycoplasma pneumoniae-induced cutaneous disease. Int J Dermatol 2009, 48:673-681.

3. Schalock PC, Brennick JB, Dinulos JGH: Mycoplasma pneumoniae infection associated with bullous erythema multiforme. J Am Acad Dermatol 2005, 52:705-706.

4. Kano Y, Mitsuyama Y, Hirahara K, Shiohara T: Mycoplasma pneumonia infection induced erythema nodosum, anaphylactoid purpura, and acute urticaria in 3 people in a single family. J Am Acad Dermatol 2007, 57:\$33-\$35.

5. Kakourou T, Drosatou P, Psychou F, Aroni K, Nicolaidou P: Erythema nodosum in children: a prospective study. J Am Acad Dermatol 2001 44:17-21.

6. Schalock PC, Dinulos JG, Pace N, Schwarzenberger K, Wenger JK: Erythema multiforme due to Mycoplasma pneumoniae infection in two children Pediatr Dermatol 2006, 23:546-555.

7. Grosber M, Alexandre M, Poszepczynska-Guigné E, Revuz J, Roujeau JC Recurrent erythema multiforme in association with recurrent Mycoplasma pneumoniae infections. J Am Acad Dermatol 2007, 56:S118-S119.

8. Said MH, Layani MP, Colon S, Faraj G, Glastre C, Cochat P: Mycoplasma pneumoniae-associated nephritis in children. Pediatr Nephrol 1999, 13:39-44.

9. Cherry JD: Anemia and mucocutaneous lesions due to Mycoplasma pneumoniae infections. Clin Infect Dis 1993, 17:S47-S51.

10. Harris JA, Kolokathis A, Campbell M, Cassell GH, Hammerschlag MR: Safety and efficacy of azithromycin in the treatment of community acquired pneumonia in children. Pediatr Infect Dis J 1998, 17:865-871.

doi:10.1186/1752-1947-6-398

Cite this article as: Shimizu et al:: Sequentially appearing erythema nodosum, erythema multiforme and Henoch-Schönlein purpura in a patient with Mycoplasma pneumoniae infection: a case report. Journal of Medical Case Reports 2012 6:398.

\section{Submit your next manuscript to BioMed Central and take full advantage of:}

- Convenient online submission

- Thorough peer review

- No space constraints or color figure charges

- Immediate publication on acceptance

- Inclusion in PubMed, CAS, Scopus and Google Scholar

- Research which is freely available for redistribution 